\title{
Decision processes in Communications Multi-path Access Systems applied within ITS
}

\author{
M. Svitek* \& T. Zelinka* \\ Faculty of Transport Sciences, Czech Technical University of Prague, 11000 Praha 1, Czech Republic \\ *Corresponding authors: svitek@fd.cvut.cz, zelinka@fd.cvut.cz
}

\begin{abstract}
The paper presents multi-path communications access systems decision processes applied within ITS (Intelligent Transport System). A competent decision process based on precisely quantified system requirements using a performance indicators tolerance range represents a critical part of the ITS access communications system. The goal of the described process is to keep the communications service continuously available with no influence on changing conditions in time and served space. Either the family of CALM standards based system or the specifically designed and configured L3/L2 switching represent relevant a solution for such a multi-path access communication system. The method of different paths service quality evaluation and the selection of the best possible active communications access path are introduced. The proposed approach is based on Kalman filtering, which separates a reasonable part of noise and also allows the prediction of the individual parameters' near future behavior. The presented classification algorithm applied to the filtered measured data combined with deterministic parameters is trained using training data, i.e., a combination of the parameters' vectors time line and relevant decisions. The classification process results are dependent on the size and quality of the training sets.
\end{abstract}

KEY WORDS: Intelligent transport systems, performance indicators, localization, navigation, multi-path access, decision processes

\section{INTRODUCTION}

The first step in addressing the ITS applications is the analysis and establishment of performance requirements on telematics applications done in co-operation with the endusers and organizations like the Railways Authority, Road and Motorways Directorates, Air Traffic Controls. The next step represents a decomposition of the systems requirements to the individual subsystems of the telematics chain.

The list of representative telematic performance indicators was developed and is widely accepted in structure (Svitek, 2005) or (Svitek \& Zelinka, 2006):

- Safety - risk analysis, risk classification, risk tolerability matrix, etc.

- Reliability - the ability to perform required functions under given conditions for a given time interval

- Availability - the ability to perform required functions at the initialization of the intended operation

- Integrity - the ability to provide timely and valid alerts to the user when a system must not be used for the intended operation 
- Continuity - the ability to perform required functions without unscheduled interruptions during the intended operation

- Accuracy - the degree of conformance between a platform's true parameters and its estimated values, etc.

A substantial part of the performance parameters analysis regarding the telematics application is represented by the decomposition of these parameters to individual subsystems of the telematics chain, including a proposal for the macro-functions of individual subsystems and information relations between macro-functions. Part of the analysis is the establishment of requirements on individual functions and information linkage so that the whole telematics chain should comply with the above defined performance parameters.

The completed decomposition of performance parameters enabled the development of a methodology for a follow-up analysis of telematics chains according to various criteria optimization of the information transfer between a mobile unit and the processing centre, maximum use of the existing information and telecommunication infrastructure, and so on.

One of the criteria appropriate for transport-telematics applications with a Global Navigation Satellite System (GNSS) is synthesis of the telematics system with minimized performance requirements on a locator, as well as a communications solution resulting in the performance parameters of the telematics application to be maintained. This synthesis does not relate only to the technical or technological part of the solution because the safeguarding of performance parameters of telematics applications is to be ensured by organizational and legislative instruments as well.

The transport telematics field deals not only with its own technologies of the ITS systems but particularly with organizational, economic, managerial and other implementing instruments of such systems, including the evaluation of the impact of ITS systems on the carriage of persons and goods, the acceptance of the approach by drivers, passengers, and the increase in the capacity of goods transport.

\section{COMMUNICATIONS SOLUTION}

\section{Telematic sub-system requirements}

Mobility of the communication solution represents one of the crucial system properties, namely in the context of, frequently, very specific system requirements.

The following communications performance indicators quantify communications service quality (Svitek \& Zelinka, 2006), (Svitek \& Zelinka, 2007) or (Zelinka \& Svitek, 2007):

- Availability

- Service Activation Time

- Mean Time to Restore (MTTR)

- Mean Time Between Failure (MTBF)

- VC availability

- Delay is an accumulative parameter and it is influenced by

- Interfaces rates

- Frame size

- Load / congestion of all in-line active nodes (switches)

- Packet/Frames Loss, and

- Security

Performance indicators applied for such communications applications must be transformable into telematic performance indicators structure and vice versa. The indicators transformability simplifies system synthesis. The additive impact of the communications performance indicators vector $\overrightarrow{t c i}$ on the vector $\Delta \overrightarrow{t m i}$ of telematic performance indicators can 
be expressed by Eq. 1, however, only under the condition that the probability levels of all studied phenomena are on the same level and all performance indicators are expressed exclusively by parameters with the same physical dimensions - in the described case in time or to time convertible variable (Svitek \& Zelinka, 2007):

$$
\Delta \overrightarrow{t m i}=T M \cdot \overrightarrow{t c i}
$$

Transformation matrix construction is dependent on the detailed communication solution and its integration into the telematic system. The probability of each phenomena appearance in the context of other processes is not deeply evaluated in the introductory period, when the specific structure of transformation matrix is identified. However, each TM element is consequently evaluated in several steps, a process based on the detailed analysis of the particular telematic and communications configuration and its appearance probability in the specific context of the whole system performance. This approach represents a subsequent iterative process managed with the goal to reach a stage where all minor indicators (relations) are eliminated and the major indicators are identified under the condition that relevant telematic performance indicators are kept within the given tolerance range.

Details of iterative method are discussed in (Svitek \& Zelinka, 2007) or (Zelinka \& Svitek, 2007). The method is designed as broadly as possible with the clear aim to be applied in the widest possible range of telematic applications. This method can also be successfully used for identification of "CALM" criteria, i.e., the tolerance range of each performance indicator, to be able to decide which alternative access technology is, in a specific time and space, evaluated as the best possible alternative.

\subsection{COMMUNICATIONS SOLUTION STRUCTURE}

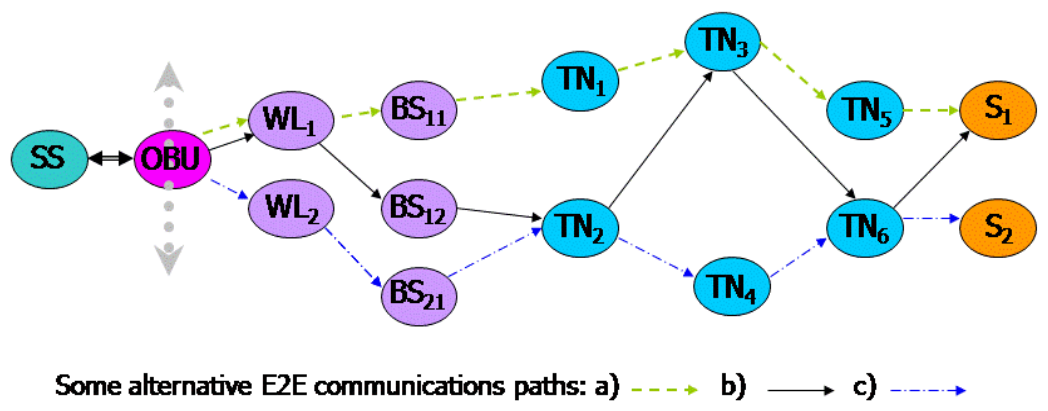

Figure 1: Telematic chain diagram

Figure 1 presents a typical telematic sub-system chain diagram (specifically the solution for the pilot project Airport (Svitek \& Zelinka, 2007) and (Zelinka \& Svitek, 2007). The outdoor unit consists of GNSS (Global Navigation Satellite System) GPS (Global Positioning System)/Galileo Sensing System (SS), On Board control and display Unit (OBU) and Wireless mobile communication units $\left(\mathrm{WL}_{\mathrm{i}}\right)$. The terrestrial communication part consists of a set of mobile cellular Base Stations $\left(\mathrm{BS}_{\mathrm{ij}}\right)$ as well as the terrestrial network based on L2 switches/nodes $\left(\mathrm{TN}_{\mathrm{i}}\right)$ interconnected with Servers $\left(\mathrm{S}_{\mathrm{i}}\right)$.

One core technology can be selected as the core solution, if possible. However, some areas need to be covered by alternative solutions. We will discuss the principles of procedures which support the selection of the best possible communications solution quantified by performance indicators and by some other parameters, e.g., service costs, as well. The technical implementation is described by the standard CALM, even though alternative solutions are also available, e.g., based on L3/L2 switching principles. 
The typical general transport telematic access solutions with the combination of a wide range of access technologies is shown on Fig. 2 (Wall, 2006).

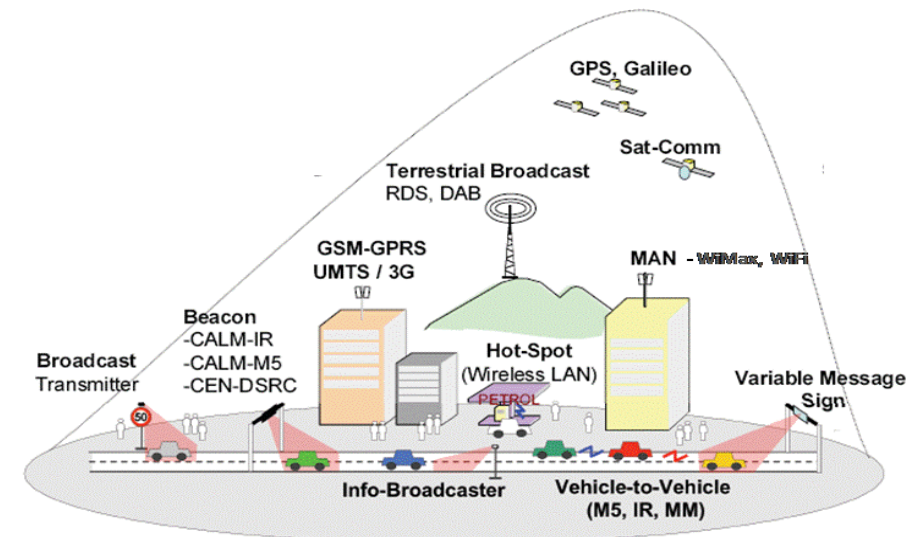

Figure 2: Transport telematics communications access solutions

\subsection{MULTI-PATH ACCESS BASED ON CALM AND L3/L2 SWITCHING.}

The family of standards ISO TC204, WG16.1 "Communications Air-interface for Long and Medium range" (CALM) represents a widely conceived concept of switching to the best available wireless access alternative in a given time and area. The substitution process of existing paths by the alternative wireless access solution is understood as the second generation of the handover principle.

Both generations of the handover action are started based on an evaluation of the performance indicators set. BER (Bit Error Rate) or packet RTD (Round Trip Delay) are typical but not the only possible performance indicators used for decision processes in data networks. Switching to the alternative path is relevant only if available tools of the lower layer are already unable to resolve performance limits. Simultaneous action on more layers can be counterproductive action.

Second generation handover action can be evoked also by the identification of a more suitable alternative, e.g., by the appearance of an alternative service with better cost conditions, even though the existing alternative is technically sufficient and safe.

An adaptive communications control system has the following architecture:

- 1st layer - Cellular Layer (CL) - represents the feed-back control processes of parameters like transmitted power, type of applied modulation, etc. The goal of processes on this layer is to keep the given set of managed parameters, for example Bit Error Rate (BER) or Round Trip Delay (RTD), within the required limits

- 2nd layer - the first generation of handover $(1 \mathrm{HL})$ - represents the seamless switching process between different cells of the same mobile network. Such an approach is applied in mobile systems like GPRS, EDGE, UMTS, Mobile WiMax (IEEE 802.16e) or WiFi (IEEE 802.11), via the amendment IEEE 802.11r. The 1HL layer shares relevant information with the CL layer (delivered usually as one system) so that there is no risk of counterproductive simultaneously operated processes on both layers - of course only in the case it is correctly designed and operated 
- 3rd layer - the second generation of handover $(2 \mathrm{HL})$ - is mostly dependent only on identification of the service performance indicators. Cellular systems are not usually designed as open systems with appropriate application inter-faces (API) so that there is mostly not a potential for the interconnection with the management of these lower layers. It is certain that the effective management of the $2 \mathrm{HL}$ layer can be reached much more easily if $1 \mathrm{HL}$ and LC layers share relevant information with the managed layer $2 \mathrm{HL}$

Communications access systems used in transport telematics are:

- Cellular systems, including 2.5G GSM and UMTS

- Mobile Wireless Broadband (MWB) with cells usually much larger than UMTS cells today namely communications systems based on IEEE Std. 802.16e and the up-coming IEEE Std. 802.20

- WiFi (IEEE 802.11 based) different alternatives - a, b, g and cellular mode option (802.11r)

- $\operatorname{DSRC}(5.8 \mathrm{GHz})$

- M5 based on standard IEEE 802.11p

- IR (Infra Read) communications solutions

- IEEE 802.15.x based solutions: Bluetooth - 15.1, UWB (Ultra Wide Band) - 15.3, ZigBee $-15.4$

- Millimeter wave technology $(62-63 \mathrm{GHz})$ used in conjunction with radar signals at similar frequencies

- Satellite communications exclusively applied for emergency and "special applications"

- W-USB (Wireless USB)

- Other media to come

Only some of presented systems have cellular architecture. In the case that the system is not cellular we can omit the 1HL layer of the presented model.

In CALM standard vertical decomposition to the individual subsystems is applied for each communications access path. Each layer can share the support of more alternative access solutions in one subsystem, when it is possible and effective. However, management remains exclusively and strictly in the horizontal layers architecture. The 1HL layer is understood only as an optional extension of L2 with no principal influence on the whole system architecture. Relevant information needed for qualified decisions is shared between layers exclusively via relevant control system structures. CALM architecture is discussed in (Wall, 2006) and (Zelinka \& Svitek, 2007). CALM applies the exclusively still not widely spread IPv6 protocol which allows, due to its extensive abilities, to continuously remotely trace active applied alternatives. Handover is accomplished in CALM exclusively on the L2 of the TCP(UDP)/IP model, i.e., out of TCP/IP competences. Handover competences given to this L2 is a suitable alternative for most of the wireless solutions.

The authors evaluated CALM an orientation as appropriate approach, however, connected with quite an extensive $R \& D$ representing a remarkable time period. As a response to the urgent need of an acceptable solution the authors proposed an alternative approach based on L3/L2 TCP/IP switching operated in a specific configuration and settings. This solution is understood as only an interim and, in functionality, limited substitution, however, with a much less demanding and therefore faster implementation. 


\section{ACCESS PATHS EVALUATION AND THE DECISION PROCESS ON POTENTIAL SEAMLESS SWITCHING TO THE ALTERNATIVE SOLUTION}

The following paragraphs describe one of the potential approaches to the decision processes, which are much less discussed than the switching approaches and their management. The proposed methodology is based on following principles:

- Measured parameters are processed by the Kalman filter. Such a process separates a reasonable part of noise and also allows for the prediction of the individual parameters' near future behavior

- A set of measured parameters extended by deterministic parameters, for example economical criteria is available together as a vector $\mathbf{x}$

- Based on time lines of vector $\mathrm{x}$, it is feasible to classify the best possible technology selection. The classification algorithm is trained using the time lines of training vectors $\mathbf{x}$ and the relevant selected paths - see for example (Svitek, 2006)

This solution does not necessarily require $2 \mathrm{HL}$ communication with the other layers, but nevertheless, it would be a much more efficient solution if such communication is at least partially possible in future implementations.

\subsection{ESTIMATION AND PREDICTION OF MEASURED PERFORMANCE DATA VECTOR P(N)}

Let us define parameter vector $\mathrm{p}(\mathrm{n})$ in the time interval $\mathrm{n}$. We will suppose that the dynamics of parameter $\mathrm{p}(\mathrm{n})$ evolute based on the following model (it is supposed that $\mathrm{p}(\mathrm{n}-1)$ is known):

$$
\boldsymbol{p}(n)=\boldsymbol{A}(n) \boldsymbol{p}(n-1)+\boldsymbol{b}(n)+\boldsymbol{q}(n)
$$

where $A(n)$ is a transition matrix, $b(n)$ is the deterministic vector of constant parameters and $\mathrm{q}(\mathrm{n})$ is the vector of Gaussian noise with the following property:

$$
\begin{aligned}
& E[\boldsymbol{q}(n)]=0 \\
& \operatorname{cov}[\boldsymbol{q}(n), \boldsymbol{q}(i)]=0 \text { for } n \neq i \\
& \operatorname{cov}[\boldsymbol{q}(n), \boldsymbol{q}(i)]=\boldsymbol{Q}(i) \text { for } n=i
\end{aligned}
$$

The equations (2) and (3) represent "the evolution form of an unknown parameters vector".

In many cases we cannot measure the vector of an unknown parameter $\mathrm{p}(\mathrm{n})$ directly, however, we can measure another vector $z(n)$ that depends on unknown parameters as follows:

$$
\boldsymbol{z}(n)=\boldsymbol{D}(n) \boldsymbol{p}(n)+\boldsymbol{r}(n)+\boldsymbol{w}(n)
$$

where $\mathrm{D}(\mathrm{n})$ is a transition matrix, $\mathrm{r}(\mathrm{n})$ is a deterministic vector of constant parameters and $\mathrm{w}(\mathrm{n})$ is the vector of Gaussian noise with the following property:

$$
\begin{aligned}
& E[\boldsymbol{W}(n)]=0 \\
& \operatorname{cov}[\boldsymbol{W}(n), \boldsymbol{w}(i)]=0 \text { for } n \neq i \\
& \operatorname{cov}[\boldsymbol{W}(n), \boldsymbol{w}(i)]=\boldsymbol{W}(i) \text { for } n=i
\end{aligned}
$$

The equations (4) and (5) represent "the evolution form of a measurement vector".

The algorithm for the estimation of a vector $\hat{\mathbf{p}}(n)_{\text {of }}$ unknown parameters together with its covariance matrix $S(n)$ can be summarized:

$$
\begin{aligned}
& \hat{\mathbf{p}}(n)=\hat{\mathbf{p}}_{e}(n)+\boldsymbol{H}(n)\left(\mathbf{z}(n)-\boldsymbol{r}(n)-\boldsymbol{D}(n) \hat{\mathbf{p}}_{e}(n)\right) \\
& \mathbf{S}(n)=\boldsymbol{S}_{e}(n)-\boldsymbol{H}(n) \boldsymbol{D}(n) \boldsymbol{S}_{e}(n)
\end{aligned}
$$


where $\hat{\mathbf{p}}_{e}(n)$ is an extrapolated estimate from the last step, $\mathbf{S}_{e}(n)$ is a covariance matrix of extrapolation and $\mathrm{H}(\mathrm{n})$ is the Kalman gain. All the mentioned parameters are possible to be recursively computed from the last estimated parameters characterized by $\hat{\mathbf{p}}(n-1), \mathbf{S}(n-1)$ according to the form:

$$
\begin{aligned}
& \hat{\boldsymbol{p}}_{e}(n)=\boldsymbol{A}(n) \hat{\boldsymbol{p}}(n-1)+\boldsymbol{b}(n) \\
& \boldsymbol{S}_{e}(n)=\boldsymbol{A}(n) \boldsymbol{S}(n-1) \boldsymbol{A}(n)^{T}+\boldsymbol{Q}(n) \\
& \boldsymbol{H}(n)=\boldsymbol{S}_{e}(n) \boldsymbol{D}(n)^{T}\left(\boldsymbol{D}(n) \boldsymbol{S}_{e}(n) \boldsymbol{D}(n)^{T}+\boldsymbol{W}(n)\right)^{-1}
\end{aligned}
$$

Equations (6) and (7) are understood as "the Kalman filtering algorithm".

Now, we suppose the non-linear evolution of an unknown parameter vector (2) and a measurement vector (4) through known non-linear functions $f($.$) and h($.$) :$

$$
\begin{gathered}
\boldsymbol{p}(n)=f(\boldsymbol{p}(n-1))+\boldsymbol{b}(n)+\boldsymbol{q}(n) \\
\boldsymbol{z}(n)=h(\boldsymbol{p}(n))+\boldsymbol{r}(n)+\boldsymbol{w}(n)
\end{gathered}
$$

The main idea is to linearize the equations (8) and (9) with the help of the first two components of Taylor series in the extrapolated value $\hat{\mathbf{p}}_{e}(n)$ (extended Kalman filtering):

$$
\begin{aligned}
& f(\boldsymbol{p}(n-1))=f\left(\hat{\boldsymbol{p}}_{\boldsymbol{e}}(n)\right)+\left.\frac{1}{2} \cdot \frac{\partial f(\mathbf{p})}{\partial \mathbf{p}}\right|_{\mathbf{p}=\hat{\mathbf{p}}_{e}(n)} \cdot\left(\boldsymbol{p}(n-1)-\hat{\boldsymbol{p}}_{\boldsymbol{e}}(n)\right) \\
& h(\boldsymbol{p}(n-1))=h\left(\hat{\boldsymbol{p}}_{\boldsymbol{e}}(n)\right)+\left.\frac{1}{2} \cdot \frac{\partial h(\mathbf{p})}{\partial \mathbf{p}}\right|_{\mathbf{p}=\hat{\mathbf{p}}_{e}(n)} \cdot\left(\boldsymbol{p}(n-1)-\hat{\boldsymbol{p}}_{\boldsymbol{e}}(n)\right)
\end{aligned}
$$

Based on the equations (10) and (11) non-linear equations (8) and (9) are transformed into a linear form and Kalman filtering could be used.

Kalman filtering can be started by the first measurement $\mathrm{z}(1)$. The initial parameters should be set up as:

$$
\begin{aligned}
& \hat{\mathbf{p}}(1)=\mathbf{H}(1)(\mathbf{z}(1)-\mathbf{r}(1)) \\
& \mathbf{H}(1)=\left(\mathbf{D}(1)^{\mathrm{T}} \mathbf{W}(1)^{-1} \mathbf{D}(1)\right)^{-1} \mathbf{D}(1)^{\mathrm{T}} \mathbf{W}(1)^{-1} \\
& \mathbf{S}(1)=\left(\mathbf{D}(1)^{\mathrm{T}} \mathbf{W}(1)^{-1} \mathbf{D}(1)\right)^{-1}
\end{aligned}
$$

\subsection{SWITCHING AS A CLASSIFICATION PROCESS}

Let us introduce the vector $\mathrm{x}$ as the vector carrying information about the values of performance parameters in a sample time. The items of vector $\mathrm{x}$ are either deterministic or random processes with the help of the Kalman filtering described above.

Let us define the classification problem as an allocation of the feature vector $\mathbf{x} \in \mathrm{R}^{\mathrm{D}}$ to one of the $\mathrm{C}$ mutually exclusive classes knowing that the class of $\mathbf{x}$ takes the value in

$$
\left\langle\Omega=\left\{\omega_{1}, \ldots \ldots ., \omega_{C}\right\}\right\rangle
$$

with probabilities $P\left(\omega_{1}\right), \ldots . ., P\left(\omega_{C}\right)$, respectively, and $\mathrm{x}$ is a realization of a random vector characterized by a conditional probability density function $p(\mathbf{x} \mid \omega), \omega \in \Omega$. This allocation means the selection of the best suited telecommunication technology based on knowledge of $\mathrm{x}$ vector.

A non-parametric estimate of the $\omega$-th class conditional density provided by the kernel method is:

$$
\hat{\mathrm{f}}(\mathbf{x} \mid \omega)=\frac{1}{\mathrm{~N}_{\omega} \cdot \mathrm{h}_{\omega}^{\mathrm{D}}} \cdot \sum_{\mathrm{i}=1}^{\mathrm{N}_{\omega}} \mathrm{K}\left(\frac{\mathbf{x}-x_{\mathrm{i}}^{\omega}}{\mathrm{h}_{\omega}}\right),
$$


where $K(.$.$) is a kernel function that integrates to one, h_{\omega}$ is a smoothing parameter for $\omega$-th class, $N_{\omega}$ stands for the sample count in class $\omega$ and $\mathbf{X}_{1}^{\omega}, \ldots ., \mathbf{x}_{N_{\omega}}^{\omega}$ is the independent training data. The density estimate defined by (13) is also called the Parzen window density estimate with the window function $K(.$.$) .$

It is a well-known fact that the choice of a particular window function is not as important as the proper selection of smoothing parameter. We use the Laplace kernel defined by the following Laplace density function:

$$
f_{L}(x ; \mu, \sigma)=\frac{1}{2 \cdot \sigma} \cdot \exp \left(-\frac{|x-\mu|}{\sigma}\right)
$$

where $x \in R, \mu \in \mathrm{R}, \sigma \in(0, \infty)$.

The product kernel is used with a vector of smoothing parameters $\boldsymbol{h}_{\omega}=\left(h_{1 \omega}, \ldots ., h_{\mathrm{D} \omega}\right)$ for each class $\omega$. The product kernel density estimate with Laplace kernel is then defined as

$$
\hat{f}(\mathbf{x} \mid \omega)=\frac{1}{N_{\omega}} \sum_{i=1}^{N_{\omega}} \prod_{j=1}^{D} \frac{1}{2 \cdot h_{\omega, j}} \exp \left(-\frac{\left|x_{j}-x_{i, j}^{\omega}\right|}{h_{\omega, j}}\right) .
$$

Smoothing vectors $\boldsymbol{h}_{\omega}$ are optimized by a pseudo-likelihood cross-validation method using the Expectation-Maximisation (EM) algorithm.

To rank the features according to their discriminative power the standard between-to within-class variance ratio is employed. This method is based on the assumption that individual features have Gaussian distributions. The feature vector $\mathbf{x} \in \mathrm{R}^{\mathrm{D}}$ takes the value to one of $\mathrm{C}$ mutually exclusive classes $\Omega=\left\{\omega_{1}, \ldots \ldots . ., \omega_{C}\right\}$. The probabilistic measure $Q_{d, i, j}\left(d, \omega_{\mathrm{i}}, \omega_{\mathrm{j}}\right)$ of two classes separability for the feature $\mathrm{d}$ (d-th component of feature vector) is defined as

$$
\mathrm{Q}_{\mathrm{d}, \mathrm{i}, \mathrm{j}}\left(\mathrm{d}, \omega_{\mathrm{i}}, \omega_{\mathrm{j}}\right)=\frac{\eta \cdot\left(\sigma_{\mathrm{i}}+\sigma_{\mathrm{j}}\right)}{\left|\mu_{\mathrm{i}}-\mu_{\mathrm{j}}\right|},
$$

where $\omega_{i}$ and $\omega_{j}$ are classes and symbol $\eta=3.0$ denotes the real constant specifying the interval taken into account (probability that the observation of a normally distributed random variable falls in $[\mu-3.0 \cdot \sigma, \mu+3.0 \cdot \sigma]$ is 0.998). The smaller the value of the measure $Q_{i, j, d}$, the better the separation of the inspected classes made by the feature $d$ is. For $\mathrm{Q}_{\mathrm{i}, \mathrm{j}, \mathrm{d}}<1$ both classes are completely separable. The measure is similar to the widely used Fisher criterion.

For multi-class problems, the two-class contributions are accumulated to get a C-class separability measure $\mathrm{Q}(\mathrm{d})$ for the feature $\mathrm{d}$ :

$$
Q(d)=\sum_{i=1}^{c} \sum_{\substack{j=1 \\ i \neq j}}^{c} Q_{d, i, j}(d, i, j) .
$$

All the features in the training data are then sorted according to their $Q(d)$ measures. The function $Q(d)$ is similar to a significance measure of the d-th component of a feature vector. The subset of $\mathrm{n}$ first features is selected as an output of this individual feature selection method. The drawback of the method is the assumption of unimodality and the fact that only linear separability is taken into account. On the other hand, the individual feature selection method based on the between-to within-class variance ratio is very fast.

The presented classification approach is effectively applicable for relevant decision processes used to select the best possible alternative access from the set of available paths. The decision is based on the evaluation of both random, as well as deterministic, processes. The introduced approach enables continuous decision processes training.

The presented method allows implementation to be started with no information flow between the layer 2HL and layers 1HL and CL. However, the proposed solution is deliberated 
to be open for future extensions in information resources to let the decision process improve by the application of potentially available information, like the status of layers 1HL and CL.

\section{CONCLUSION}

The main goal of our research is to introduce a new generation of Intelligent Transport Services (ITS) which can be continuously available (on a defined probability level). Due to the regular complexity of areas covered by telematic services we have concentrated on the wireless access solution designed as seamless switched combination of more independent access solutions, i.e., a multi-path access system.

The process of access solution switching has been the subject of intensive R\&D and different approaches have already been published. One of alternatives $-\mathrm{a}$ family of standards CALM - represents a promising response to ITS requirements. However, due to the complexity of the proposed solution it is inevitable that a quite remarkable amount of time to resolve all issues can be expected. The proposed alternative approach, based on L3/L2 TCP/IP switching operated under a specific configuration and settings, is understood only as a potential interim and, in functionality, a limited substitution, however, with much less demanding and therefore faster implementation conditions compared, for example, to the ones of CALM.

The method of different paths evaluation and the decision process background has not been as widely discussed as the core switching alternatives. One of the possible approaches was studied and the core principles of the proposed solution are presented.

The measured parameters of all available alternative access paths are processed by the Kalman filter with the aim of separating a reasonable part of the data noise. The Kalman filter also allows for the prediction of the individual parameters' near future behavior. The filtered flow of the measured parameters vectors can then be extended by deterministic parameters, for example the economical criteria. The resultant vector $\mathbf{x}$ time line allows the classification of the best possible technology selection from those for which the relevant time line of vectors $\mathbf{x}$ is available. The classification algorithm is based on the training procedure using relevant training data - i.e. a line of training vectors $\mathbf{x}$ and relevant to data selected paths. Due to the fact that only linear separability is taken into account, the individual feature selection method based on the between-to within-class variance ratio represents a very swift approach.

The presented classification approach is applicable for relevant decision processes on the top layer of the communications system management to select the best possible access alternative from the set of available paths. The decision is based on an evaluation of both random, as well as deterministic processes, and the introduced approach enables continuous decision processes training, as well as the future information resources extension obtained namely from potentially available lower layers of the multilayer adaptive communications management system.

\section{REFERENCES}

Svitek, M., 2005. Architecture of ITS Systems and Services in the Czech Republic. International Conference Smart Moving 2005, Birmingham, England.

Svitek, M., 2005. Intelligent Transport Systems - Architecture, Design methodology and Practical Implementation. Key-note lesson, 5th WSEAS/IASME Int. Conf. on Systems Theory and Scientific Computation, Malta. 
Svitek, M., Zelinka, T., 2006. Communications Tools for Intelligent Transport Systems. Proceedings of 10th WSEAS International Conference on Communications, Athens, pp 519 522ISSN 1790-5117, ISBN 960-8457-47-5.

Svitek, M., Zelinka, T., 2006. Communications Solutions for ITS Telematic Subsystems. WSEAS Transactions on Business and Economics, Issue 4 (2006), Vol. 3, Athens, pp 361 367, ISSN 1109-9526.

Svitek, M., Zelinka, T., 2006. Telecommunications solutions for ITS. Towards Common Engineering \&Technology for Land, Maritime, air and Space Transportation - ITCT 2006, CNISF, Paris, pp 69-70.

Svitek, M., Zelinka, T., 2007. Communication solution for GPS based airport service vehicles navigation. EATIS'97 ACM-DL Proceedings, Faro (Portugal), ISBN \#978-1-59593598-4.

Svitek, M., Zelinka, T., 2007. Communication solution for Vehicles Navigation on the Airport territory. Proceedings of the 2007 IEEE Intelligent Vehicle Symposium, Istanbul, Turkey, pp 528-534, IEEE Catalogue number 07TH8947, ISBN 1-4244-1068-1.

Svitek, M., Zelinka, T., 2007. Communications Environment for Telematic Subsystems. Proceedings of 11-th World Multi-Conference on Systemic, Cybernetics and Informatics, Volume II, IIIS/IFSR, Orlando, FL, pp 362-367, ISBN-10: 1-934272-16-7, ISBN-13: 978-1934272-16-9.

Svitek, M., Zelinka, T., 2007. Communications Challenges of the Airport Over-ground Traffic Management. Proceedings of the 11th WSEAS International Multi-conference CSCC, Volume - Advances in Communications, Agion Nikolaos, Crete Island, Greece, pp. 228 234, ISSN 1790-5117, ISBN 978-969-8457-91-1.

Zelinka, T., Svitek, M., 2007. Communications Scheme for Airport Service Vehicles Navigation. Proceedings of International Conference TRANSTEC Prague, Czech Technical University, Faculty of Transport Science and University of California, Santa Barbara, Praha, pp. 160 - 166, ISBN 978-80-01-03782-9.

Zelinka, T., Svitek, M., 2007. Communication Scheme of Airport Over-ground Traffic Navigation System. Proceedings of the International Symposium on Communications and Information Technologies - ISCIT 2007. IEEE Sydney, IEEE Catalogue No. 07EX1682(C), ISBN 1-4244-977-2, Library of Congress 2007920360.

Wall, N., 2006. CALM - why ITS needs it. ITSS 6 (September).

Zelinka, T., Svitek, M., 2006. CALM - Telecommunication Environoment for Transport Telematics. Technology \& Prosperity, Vol. XI, special edition (Nov./06), ISSN 1213-7162.

Svitek, M., 2006. Dynamical Systems with Reduced Dimensionality. Neural Network World edition, II ASCR and CTU FTS, Praha, ISBN:80-903298-6-1, EAN: 978-80-903298-6-7. 